\section{Evaluation of octreotide to assess insulin- mediated glucose disposal by the insulin suppression test}

Dear Sir,

Insulin resistance is commonly seen in patients with impaired glucose tolerance or non-insulin-dependent diabetes mellitus NIDDM, normoglycaemic first-degree relatives of patients with NIDDM, patients with high blood pressure and their normotensive first-degree relatives, dyslipidaemic individuals with high plasma triglyceride and low HDL cholesterol concentrations, and both microvascular angina and conventional coronary heart disease [1-8]. Given these observations, it is not surprising that this phenomenon has received, and continues to receive considerable attention. In this context, plasma insulin concentration is often used as a surrogate marker for insulin-mediated glucose uptake. Although the relationship between plasma insulin concentration and insulin resistance is statistically significant in non-diabetic subjects [9], measurement of the plasma insulin level is not identical to the direct determination of insulin resistance, either quantitatively or conceptually.

For approximately 20 years we have used a simple infusion procedure (insulin suppression test) to directly measure insulinmediated glucose uptake based upon the suppression of endogenous insulin secretion and the constant infusion of glucose and exogenous insulin [1-8]. Under these conditions, similar steady-state plasma insulin (SSPI) concentrations are reached in all individuals, and the steady-state plasma glucose (SSPG) concentration provides a direct estimate of insulin-mediated glucose uptake. More recently we have used somatostatin to suppress endogenous insulin secretion as initially suggested by Harano et al. [10]. Although this approach is satisfactory, it suffers from some practical drawbacks. For example, in the United States the use of somatostatin requires filing for an investigative new drug number at the Food and Drug Administration. A specific grade of good manufacturing practice of this material must be purchased in bulk and prepared in aliquots for intravenous infusion. Furthermore, it is necessary to prove lack of pyrogenicity for these preparations. The present study was initiated in an effort to overcome these practical hurdles, and to simplify the use of the insulin suppression test to directly assess insulin resistance. Specifically, we compared the results of measuring insulin resistance with the commercially available somatostatin analogue, octreotide, and somatostatin.

The study population consisted of 29 normal volunteers, 13 males and 16 females in good general health, taking no medication known to influence carbohydrate or lipid metabolism. They had a mean age of 46 years (range 22-69) and a mean body mass index of $26.1 \mathrm{~kg} / \mathrm{m}^{2}$ (range 18.6-35.4). Criteria for inclusion into the study included a normal medical history, physical examination, haemogram, and chemical screening battery. In addition, all subjects had a normal oral glucose tolerance test. This study was approved by the Stanford Human Subjects Committee, and each subject gave written informed consent.

The insulin suppression test (IST) was performed in each volunteer on two occasions, between 2 days and 2 months apart. Volunteers were instructed not to change their usual diet or level of physical activity during the interval between the two tests, and there was no significant difference in their weight on the two oc-

Corresponding author: G. M. Reaven, MD, Division of Gerontology, VA Medical Center (182-B), 3801 Miranda Avenue, Palo Alto, CA 94304 USA casions. In 18 subjjects, one IST was performed with somatostatin (SRIF) and the other with octreotide (a gift from Dr. B. Given, Sandoz Research Institute, East HANOVER, NJ), with the order of the two tests being randomized. In 11 subjects the IST was performed with SRIF on two occasions.

Volunteers were admitted to the Stanford General Clinical Research Center on the afternoon before each study. The IST was performed at 08.00 hours following at 12 -h fast. All patients were infused with glucose $\left(240 \mathrm{mg} \cdot \mathrm{m}^{2} \cdot \mathrm{min}^{-1}\right)$ and insulin $\left(25 \mathrm{mU} \cdot \mathrm{m}^{2} \mathrm{~min}^{-1}\right)$ for $180 \mathrm{~min}$ via an indwelling catheter in a superficial antecubital vein. In addition, either SRIF ( $5 \mu \mathrm{g} / \mathrm{min})$ or octreotide $(0.5 \mu \mathrm{g} / \mathrm{min}$ preceded by a $25 \mu \mathrm{g}$ bolus $)$ was infused over the same time period. Venous blood samples were obtained for measurement of plasma glucose and insulin concentrations from a similar catheter inserted in a contralateral antecubital vein kept patent by slow infusion of $0.9 \% \mathrm{NaCl}[3-$ 8]. Blood was drawn every $30 \mathrm{~min}$ until $150 \mathrm{~min}$ and then every 10 min from $150-180 \mathrm{~min}$. The mean value of these last four measurements was used to calculate SSPG and SSPI concentrations. Since the SSPI levels are comparable in all subjects, the height of the SSPG response is a direct reflection of the efficiency of insulin-mediated glucose disposal. Thus, the higher the SSPG value the more insulin resistant the subject. Data are expressed as the mean ( \pm SEM), and the significance of the difference in SSPG and SSPI responses observed between the two ISTs was evaluated by two-way (mean values) or three-way (values from multiple time points) analysis of variance (ANOVA) with "difference" and "patient", and/or time points being the factors.

A comparison of the time course of plasma insulin and glucose concentrations when the IST was performed with either SRIF or octreotide is shown in Figure $1 \mathrm{~A}$. It is apparent that the values for both insulin and glucose were essentially identical throughout the two studies, irrespective of whether SRIF or octreotide was used to suppress endogenous insulin secretion. In addition, mean values of the last four time points for the SSPI $(349 \pm 36$ vs $347 \pm 27 \mathrm{pmol} / 1)$ and SSPG $(7.1 \pm 0.8$ vs $6.9 \pm$ $0.7 \mathrm{mmol} / \mathrm{l})$ concentrations were the same when subjects were infused with octreotide or SRIF.

Individual SSPG values for the 18 subjects infused with both SRIF and octreotide are shown in Figure $1 \mathrm{~B}$ as a scattergram. These results indicate that SSPG values resulting from the two IST studies were highly correlated $(r=0.93, p<0.001)$ over an approximate eight-fold range of SSPG concentrations.

Figure $1 \mathrm{C}$ displays the relationship observed when the IST was performed in 11 individuals, using SRIF on both occasions to suppress endogenous insulin secretion. As was the case in Figure $1 \mathrm{~B}$, the SSPG values resulting from the two tests were highly correlated $(r=0.97, p<0.001)$. Not surprisingly, SSPI (402 \pm 39 vs $406 \pm 51 \mathrm{pmol} / \mathrm{l})$ and $\operatorname{SSPG}(9.3 \pm 1.6$ vs $9.4 \pm$ $1.6 \mathrm{mmol} / \mathrm{l}$ ) concentrations in the two studies were essentially identical.

The data shown in Figures $1 \mathrm{~A}$ and $\mathrm{B}$ clearly demonstrate that the SSPI and SSPG values achieved during the IST are similar, irrespective of the use of SRIF or octreotide to suppress endogenous insulin. Furthermore, this was true of both the time course and the final steady-state plasma glucose and insulin concentrations. Consequently, the IST can be successfully performed with octreotide, enabling the interested clinical investigator to assess insulin-mediated glucose disposal in humans without the inconvenience of having to obtain an investigative new drug number and be responsible for assuring the sterility and lack of pyrogenicity of the material used to suppress endogenous insulin secretion.

The data presented in Figures $1 \mathrm{~B}$ and $\mathrm{C}$ provide additional evidence of the reproducibility of the SSPG values obtained when the IST is performed in the same individual on two occa- 

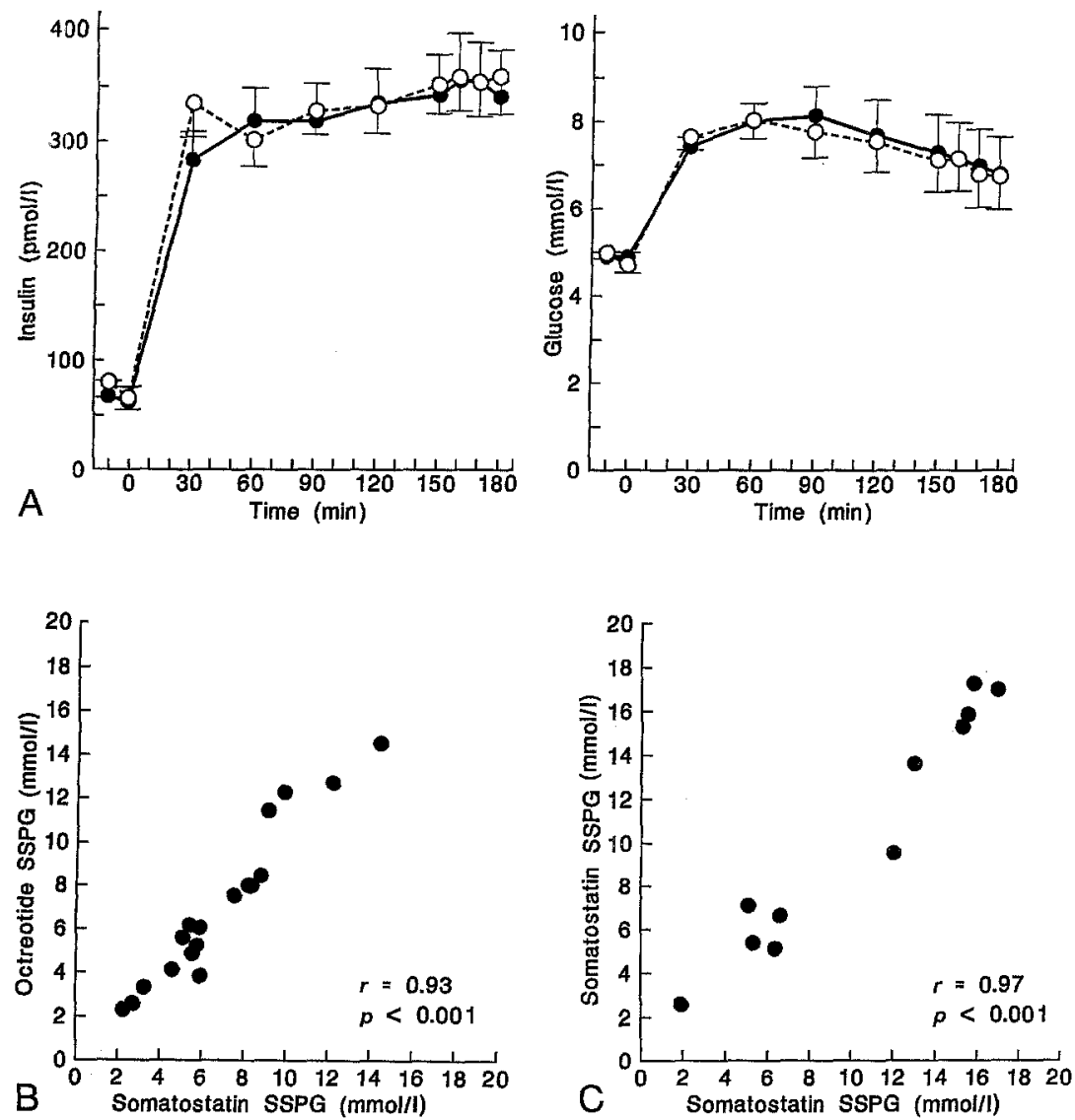

Fig.1A-C. A Time course of plasma insulin and glucose concentrations achieved during the insulin suppression test, and B correlation between steady-state plasma glucose (SSPG) values, achieved using either somatostatin ( ) or octreotide to suppress endogenous insulin. C Reproducibility of SSPG achieved when the insulin suppression test was performed on two occasions using somatostatin

sions. Specifically, the correlation coefficients between the two tests were $r=0.93$ and $r=0.97$ in duplicate studies in the 18 and 11 subjects, respectively. It should be emphasized that the SSPG concentrations of the individuals studied varied over an eight-fold range, indicating that the IST can provide reproducible estimates of insulin-mediated glucose uptake irrespective of whether the subjects are insulin sensitive or insulin resistant.

Finally, the results of these studies emphasize the utility of the IST to compare insulin-mediated glucose disposal in nondiabetic subjects. Interest in the role played by insulin resistance in the pathogenesis and clinical courses of several diseases has increased considerably. Plasma insulin concentration is often used as a surrogate marker for insulin resistance. However, individuals can vary in both the hepatic and peripheral catabolism of insulin, and the presence of hyperinsulinaemia cannot necessarily be equated with being synonymous with insulin resistance. Consequently, the view that quantitative differences in plasma insulin level can provide accurate estimates of insulin-mediated glucose uptake is problematic. In this context, use of the IST is worthy of consideration.

We have previously shown in a large population of nonobese and obese individuals with varying degrees of glucose tolerance that values of insulin resistance obtained with the IST are highly correlated $(r=0.93)$ with those achieved with the glucose clamp technique [11]. Thus, the results of the present study in non-diabetic subjects are also likely to apply to patients with states of glucose intolerance. The IST is highly reproducible, as both this and the earlier study [11] demonstrated, and the results are not affected by investigator behaviour. Finally, it is easily and safely performed, less investigator intensive, requiring only a 180-min infusion, with plasma glucose and insulin concentrations measured on four occasions during the final $30 \mathrm{~min}$, and it is possible to obtain values for insulin-mediated glucose uptake without the need for sophisticated mathematical modelling. As such, the IST using octreotide to inhibit endogenous insulin secretion provides the investigator with a simple, practical and time-tested approach to quantify insulin-mediated glucose disposal in human beings.

D. Pei, C. N. O.Jones, R. Bhargava, Y.-D.I.Chen, G. M. Reaven

\section{References}

1. Shen S-W, Reaven GM, Farquhar JW (1970) Comparison of impedance to insulin mediated glucose uptake in normal and diabetic subjects. J Clin Invest 49: 2151-2160

2. Ginsberg H, Kimmerling G, Olefsky JM, Reaven GM (1975) Demonstration of insulin resistance in untreated adult onset diabetic subjects with fasting hyperglycemia. J Clin Invest 55: 454-461

3. Laws A, Stefanick ML, Reaven GM (1989) Insulin resistance and hypertriglyceridemia in nondiabetic relatives of patients with noninsulin-dependent diabetes mellitus. J Clin Endocrinol Metab 69: 343-347

4. Shen D-C, Shieh S-M, Fuh M-T, Wu D-A, Chen Y-DI, Reaven GM (1988) Resistance to insulin-stimulated-glucose uptake in patients with hypertension. $\mathrm{J}$ Clin Endocrinol Metab 66: 580-583

5. Facchini F, Chen Y-DI, Clinkingbeard C, Jeppesen J, Reaven GM (1992) Insulin resistance, hyperinsulinemia and dyslipidemia in non-obese individuals with a family history of hypertension. Am J Hypertens 5: 694-699

6. Laws A, Reaven GM (1992) Evidence for an independent relationship between insulin resistance and fasting plasma 
HDL-cholesterol, triglyceride and insulin concentrations. J Int Med 231: 25-30

7. Fuh MT-M, Jeng C-Y, Mason MS-S, Sheu WH-H, Chen YDI, Reaven GM (1993) Insulin resistance, glucose intolerance, and hyperinsulinemia in patients with microvascular angina. Metabolism 42: 1090-1092

8. Young MH, Jeng C-Y, Sheu WH-Het al. (1993) Insulin resistance, glucose intolerance, hyperinsulinemia and dyslipidemia in patients with angiographically demonstrated coronary artery disease. Am J Cardio 72: 458-460

9. Hollenbeck CB, Chen N, Chen Y-DI, Reaven GM (1984) Relationship between the plasma insulin response to oral

\section{Selection of control subjects for case/control analysis of susceptibility to Type 1 (insulin-dependent) diabetes mellitus}

\section{Dear Sir,}

Pozzilli et al. [1] suggest several possible sources for control subjects for studies of Type 1 (insulin-dependent) diabetes. For the examination of HLA-associations alone they suggest that blood donors are likely to be satisfactory, subject to adequate matching by ethnic group, which seems a very sensible and practical proposal if the donors' ethnicity is adequately known, an if the cases are obtained from the entire catchment area of the blood donor service.

They suggest, for studies looking at environmental factors, a number of additional sources for control subjects. They are of the view that individuals who may later develop diabetes should be excluded, and that various efforts should be made to match diabetic cases and control subjects for some of the known HLA associations.

We do not agree with this proposal. The purpose of matching in analytical epidemiological studies of environmental determinants of disease is quite different, and has nothing to do with the fundamental design issue of collecting diabetic cases and sampling control subjects in an unbiased way. Matching is essentially a convenience, and should be used for clearly identified, and easily measured risk factors, such as age and sex, which are not in themselves of interest as analytical variables. Briefly, matching induces a confounding of the disease-exposure relationship by the matching factors, and this confounding is adjusted for in the analysis. A consequence of this is that it is not possible to carry out any meaningful analyses using the matching factors. Indeed matching by HLA phenotypes would exclude the possibility of investigating the interaction between environmental exposures and genetic susceptibility.

The most significant development in epidemiologic methodology in the last decade is a more detailed understanding of the nature of the case-control study. Miettinen [2] has summarised this work, albeit rather opaquely. Rothman [3] is a more accessible source. The key notion is the unbiased comparison of a census of cases with a sample from a base of control subjects. This base can be most elegantly defined as "all those persons

Corresponding author: Dr. A. Staines, LRF Unit for Clinical Epidemiology, 17 Springfield Mount, University of Leeds, Leeds LS2 9NG, UK glucose and insulin stimulated glucose utilization in normal subjects. Diabetes 33: 460-463

10. Harano Y, Hidaka H, Takatsuki S et al. (1978) Glucose, insulin and somatostatin infusion for the determination of insulin sensitivity in vivo. Metabolism 27: 1449-1452

11. Greenfield MS, Doberne L, Kraemer F, Tobey T, Reaven GM (1981) Assessment of insulin resistance with the insulin suppression test and the euglycemic clamp. Diabetes 30 : 387392

who, if they were to develop the disease of interest during the study period, would be eligible to be cases" [3]

The choice of control subjects is critical. It is not difficult to match cases and control subjects too closely, and one of the proposals, selecting control subjects from classmates, would almost certainly accomplish this. There is good evidence [4] that factors acting in early life are important in the aetiology of childhood diabetes, and some evidence [5, and Staines, Bodansky, McKinney et al. unpublished observations] that area of residence is a significant risk factor for Type 1 diabetes. Selection of classmates would almost certainly match closely on residence, and might well match closely for social factors, which are related to infant feeding and child rearing practices. The potential influence of these factors could be entirely obscured.

Eliminating persons with a family history of diabetes from consideration as control subjects would tend to selectively eliminate families exposed to the putative environmental risk factors, and could therefore tend to exaggerate any associations which might be uncovered. It would also tend to strengthen any confounding present in the study population, and would render the entire analysis suspect. In additon the difficulty of implementing this in practice, and incurring a significant extra cost in approaching control subjects should not be under-estimated. A more appropriate approach would be to record information on family history, and to adjust for this in the analysis.

We agree that selecting two haplotype different siblings of diabetic patients as control subjects would cause serious problems of interpretation, both for the analysis of genetic risks, and for the analysis of environmental factors.

In conclusion the choice of appropriate control subjects for a case-control study is certainly not easy. Control subjects and diabetic cases should have precisely the same eligibility criteria, and control subjects should be sampled from the best available population lists, if these exist. If they don't, great care needs to be taken to avoid bias and overmatching of diabetic cases and control subjects. For case-control studies looking at environmental risk factors, (as opposed to cohort studies) it is hard to see any justification for genetic exclusion criteria applied to control subjects alone.

Yours sincerely,

A. Staines,

P. A. McKinney

\section{References}

1. Pozzilli P, Buzzetti R, Bottazzo GF, Tosi R (1993) The selection of control subjects for case/control analysis of susceptibility to type 1 (insulin-dependent) diabetes mellitus. Diabetologia 36: 1208-1209 